\title{
Prevalência e fatores agravantes do sintoma de refluxo gastroesofágico em estudantes de medicina da Universidade Federal do Tocantins
}

\author{
Prevalence and aggravating factors of symptom of gastroesophageal reflux in medical \\ students of the Federal University of Tocantins
}

Laís de Souza Meireles ${ }^{1,4}$, Bethania Luciana dos Santos Holanda ${ }^{1,4}$, Milena Aguiar de Faria ${ }^{1,4}$, Danilo Lopes Castro ${ }^{1,4}$, Itágores Hoffman I. Lopes Sousa Coutinho ${ }^{2,4}$, Talita Buttarello Mucari ${ }^{3,4}$

${ }^{1}$ Acadêmicos do Curso de Medicina; ${ }^{2}$ Professor Assistente do Curso de Medicina; ${ }^{3}$ Professora Adjunta do Curso de Medicina; ${ }^{4}$ Universidade Federal do Tocantins (UFT), Palmas, TO.

\section{RESUMO}

Objetivos: Avaliar a prevalência e fatores agravantes do sintoma de pirose possivelmente associado a refluxo gastroesofágico, em estudantes de medicina da Universidade Federal do Tocantins (UFT).

Métodos: Um estudo transversal avaliou questionários aplicados a estudantes do Curso de Medicina da Universidade Federal do Tocantins entre 23/09/2013 e 04/10/2013. As variáveis analisadas foram faixa etária, sexo, índice de massa corporal, frequência de ocorrência dos sintomas, percepção de fatores que agravam o sintoma de pirose, tabagismo e uso de medicamentos para aliviar sintomas de pirose. Para calcular a prevalência, foi considerado como sintoma de refluxo gastroesofágico a ocorrência de pirose mais de uma vez por semana, mas os fatores agravantes foram pesquisados nos indivíduos que referiam ter tido o sintoma pelo menos uma vez na vida. A análise estatística incluiu as frequências absolutas e relativas das variáveis, bem como os intervalos de confiança de $95 \%$.

Resultados: Foram avaliados 186 estudantes, sendo que $160(86,0 \%)$ referiram ter tido pirose em alguma ocasião. Naqueles que referiram tal sintoma, a faixa etária predominante foi de 21 a 25 anos $(65,0 \%)$, sendo $51,3 \%$ do sexo masculino. O sintoma de pirose ocorrendo pelo menos uma vez por semana foi referido por $88(47,3 \%)$ dos entrevistados, sendo uma vez por semana por $53(28,5 \%)$ e mais de uma vez por semana por 35 (18,8\%). Considerando todos os 160 estudantes que referiam ter tido pirose em alguma ocasião, constatou-se piora do sintoma na presença de alterações emocionais (67,5\%), ingestão de alimentos gordurosos ou condimentados $(63,7 \%)$ e bebidas alcoólicas (60,6\%).

Conclusões: Os estudantes de medicina em estudo apresentaram uma alta prevalência do sintoma pirose. O sintoma era agravado por ingestão de alimentos gordurosos ou condimentados, alterações emocionais e bebidas alcoólicas.

DESCRITORES: DOENÇA DO REFLUXO GASTROESOFÁGICO; PIROSE; HÁBITOS ALIMENTARES; SINTOMA; ESTUDANTES DE MEDICINA; TENSÃO EMOCIONAL.

\section{ABSTRACT}

Aims: To evaluate the prevalence and aggravating factors of heartburn symptom possibly associated with gastroesophageal reflux in medical students of the Federal University of Tocantins.

Methods: A cross-sectional study evaluated questionnaires given to students of the Medical School of the Federal University of Tocantins between 09/23/2013 and 10/04/2013. The analyzed variables were age, sex, body mass index, frequency of occurrence of symptoms, perception of factors that aggravate the symptom of heartburn, smoking, and use of drugs to relieve symptoms of heartburn. To calculate the prevalence, occurrence of heartburn more than once a week was considered as a symptom of gastroesophageal reflux, but the aggravating factors were investigated in subjects who reported having had this symptom at least once in life. Statistical analysis included absolute and relative frequencies of the variables as well as the confidence intervals of $95 \%$.

Results: At all 186 students were evaluated, of which 160 reported heartburn at some time. In those who reported such symptom, the predominant age group was 21-25 years (65.0\%), and 51.3\% were male. The symptom of heartburn occurring at least once a week was reported for $88(47.3 \%)$ of respondents, being $53(28.5 \%)$ for once a week and $35(18.8 \%)$ for more than once a week. Considering all the 160 students who reported having had heartburn at some time, there was a worsening of symptoms in the presence of emotional disorders (67.5\%), eating fatty foods $(63.7 \%)$ and alcoholic beverages $(60.6 \%)$.

Conclusions: Medical students in the study showed a high prevalence of heartburn. This symptom was aggravated in most interviewees by intake of fatty or spicy foods, emotional changes, and alcoholic beverages.

KEY WORDS: GASTROESPHAGEAL REFLUX DISEASE; HEARTBURN; FOOD HABITS; SYMPTOM; MEDICAL STUDENTS; EMOTIONAL TENSION. 


\section{INTRODUÇÃO}

A doença do refluxo gastroesofágico (DRGE) apresenta uma incidência em ascensão e é considerada uma das afecções do trato digestório de maior prevalência nos países ocidentais. ${ }^{1}$ Estima-se que a prevalência na população adulta do Brasil seja de $12 \% .^{2}$

O refluxo gastroesofágico, processo fisiológico que pode acontecer ao longo do dia, consiste na passagem involuntária de conteúdos gástricos para o esôfago. ${ }^{3}$ Já a DRGE, segundo o Consenso Brasileiro da DRGE, é uma afecção crônica decorrente do fluxo retrógrado do conteúdo gastroduodenal para o esôfago e/ou órgãos adjacentes a ele, propiciando ao desenvolvimento de sintomas e/ou sinais esofagianos e/ou extraesofagianos, associados ou não a lesões teciduais. ${ }^{4}$

Assim como outras doenças sistêmicas, a DRGE interfere na qualidade de vida dos pacientes, podendo até influenciar na produtividade tanto do trabalho quanto das atividades diárias. É notória a relevância do diagnóstico precoce e tratamento adequado da doença, visto que suas complicações, particularmente o potencial maligno da exposição prolongada ao conteúdo ácido proveniente do estômago, podendo ocasionar esofagite de refluxo e modificações nas células do epitélio gástrico, podem agravar o estado de saúde do individuo. No entanto, devido à falta de diagnóstico precoce e prática de automedicação, é difícil estimar a real prevalência dessa enfermidade. ${ }^{5-7}$

A queimação que se estende do manúbrio esternal até a base do pescoço é chamada popularmente de azia e em termos médicos de pirose. Sua duração e frequência são preditores da gravidade da esofagite e podem indicar a presença da DRGE. ${ }^{8}$

A pirose é um marcador com alta especificidade e valor preditivo positivo para o diagnóstico dessa patologia. ${ }^{7}$ Sua identificação é aceita como marcador clínico da DRGE quando tiver ocorrido uma ou mais vezes por semana nos últimos 12 meses, ou duas vezes por semana nas últimas quatro a oito semanas. ${ }^{9,10}$ Vários pacientes podem apresentar ainda epigastralgia e dispepsia. ${ }^{11}$ Além das manifestações típicas (pirose e/ou regurgitação), podem ocorrer também as manifestações atípicas (sintomas crônicos laríngeos e asma). Estas tornam a suspeita diagnóstica mais difícil e demorada.?

Apesar da crescente importância do diagnóstico precoce da DRGE em termos clínicos, terapêuticos e econômicos, existem poucos estudos disponíveis sobre a prevalência da doença e o perfil dos pacientes. ${ }^{8} \mathrm{Em}$ virtude da carência de dados epidemiológicos locais sobre a DRGE, bem como seu impacto negativo na qualidade de vida, aumento dos custos com saúde e risco para adenocarcinoma do esôfago, o objetivo deste estudo foi acrescentar dados epidemiológicos sobre a prevalência e fatores agravantes do sintoma de pirose e de possível DRGE em estudantes de medicina da Universidade Federal do Tocantins.

\section{MÉTODOS}

Foi realizado um estudo transversal através da análise de um questionário criado pelos próprios autores, aplicado aos estudantes do Curso de Medicina da Universidade Federal do Tocantins (UFT), Campus de Palmas. O estudo foi aprovado pelo Comitê de Ética em Pesquisa desta instituição, pelo parecer consubstanciado 159/2013.

Foram incluídos apenas os estudantes com idade superior a 18 anos e que aceitaram participar da pesquisa através da assinatura do Termo de Consentimento Livre e Esclarecido, no período de 23/09/2013 a 04/10/2013. Não foram incluídos os estudantes que estavam em ambiente de estágio (Internato), que corresponde aos dois últimos anos do curso, e aqueles que por outros motivos não estavam presentes no Campus durante a aplicação dos questionários.

As variáveis analisadas foram faixa etária, sexo, índice de massa corporal (IMC), frequência de ocorrência do sintoma, percepção de fatores que agravam o sintoma de pirose, tabagismo e uso de medicamentos para aliviar o sintoma de pirose. Foi considerado como sintoma de refluxo gastroesofágico a ocorrência de pirose mais de uma vez por semana.

Os resultados foram analisados através do programa Epi Info 3.5.1, sendo calculadas as frequências absolutas e relativas, bem como os intervalos de confiança (IC) de 95\%.

\section{RESULTADOS}

Os estudantes do Curso de Medicina da UFT, na época da pesquisa, totalizavam 320, entre os quais 186 atenderam aos critérios de inclusão e responderam aos questionários. Da amostra de 186 estudantes que participaram da pesquisa, $160(86 \%)$ relataram apresentar pirose em alguma ocasião, enquanto 26 (14\%) negaram tal sintoma. O sintoma de pirose ocorrendo pelo menos uma vez por semana foi referido por $88(47,3 \%)$ dos entrevistados, sendo uma vez por semana por $53(28,5 \%)$ e mais de uma vez por semana por $35(18,8 \%)$. Noventa e oito $(52,7 \%)$ estudantes 
referiam o sintoma menos de uma vez por semana ou nunca (Tabela 1).

Tabela 1. Levantamento do sintoma de pirose e das frequências de ocorrência do sintoma em 186 estudantes de medicina da Universidade Federal do Tocantins. Palmas, TO, Brasil, 2013.

\begin{tabular}{lccr}
\multicolumn{1}{|c}{ Variáveis } & N & $\%$ & IC $\mathbf{9 5 \%}$ \\
\hline $\begin{array}{l}\text { Sensação de pirose em alguma ocasião } \\
\text { Sim }\end{array}$ & 160 & 88 & $9,3-19,8$ \\
$\quad$ Não & 26 & 14 & $80,2-90,7$ \\
\hline Frequência de pirose & & & \\
$\quad$ Mais de uma vez por semana & 35 & 18,8 & $13,5-25,2$ \\
$\quad$ Uma vez por semana & 53 & 28,5 & $22,1-35,6$ \\
$\quad$ Menos de uma vez por semana ou nunca & 98 & 52,7 & $45,3-60,0$ \\
\hline
\end{tabular}

IC = Intervalo de confiança.

Entre os 160 indivíduos que referiram o sintoma pirose, a faixa etária predominante foi de 21-25 anos, correspondendo a 104 estudantes $(65,0 \%)$. Em relação ao sexo, observou-se equilíbrio, com um total de $82(51,3 \%)$ do sexo masculino. Ao analisar o índice de massa corporal (IMC) da amostra observou-se predominância de um padrão dentro da normalidade, com IMC entre 21-24,9, correspondendo a 73 (45,6\%) entrevistados.

Entre os 160 entrevistados que relataram ter tido pirose em alguma ocasião, $108(67,5 \%)$ relataram piora deste sintoma devido a alterações emocionais, $102(63,7 \%)$ por ingestão de alimentos gordurosos ou condimentados e $97(60,6 \%)$ em decorrência da ingestão de bebidas alcoólicas (Tabela 2). Dos sintomáticos, 51 (31,9\%) faziam uso de medicação contínua para aliviar os sintomas. A maioria, $156(97,5 \%)$ não era tabagista.

Tabela 2. Distribuição de frequências dos fatores agravantes de pirose em 160 estudantes do Curso de Medicina da Universidade Federal do Tocantins, que referiram ter tido o sintoma em alguma ocasião. Palmas, TO, Brasil, 2013.

\begin{tabular}{|lccc|}
\hline \multicolumn{1}{|c}{ Fatores agravantes para pirose } & N & $\%$ & IC $\mathbf{9 5 \%}$ \\
\hline Alimentos gordurosos ou condimentados & & & \\
$\quad$ Aumenta muito & 70 & 43,8 & $35,9-51,8$ \\
Aumenta pouco & 32 & 20,0 & $14,1-27,0$ \\
$\quad$ Não aumenta & 58 & 36,3 & $28,8-44,2$ \\
\hline Bebidas alcoólicas & & & \\
$\quad$ Aumenta muito & 37 & 23,1 & $16,8-30,4$ \\
$\quad$ Aumenta pouco & 60 & 37,5 & $30,0-45,5$ \\
$\quad$ Não aumenta & 63 & 39,4 & $31,8-47,4$ \\
Questões emocionais & & & \\
$\quad$ Aumenta muito & 47 & 29,4 & $22,4-37,1$ \\
$\quad$ Aumenta pouco & 61 & 38,1 & $30,6-46,1$ \\
$\quad$ Não aumenta & 52 & 32,5 & $25,3-40,3$ \\
\hline
\end{tabular}

$\mathrm{IC}=$ Intervalo de confiança.

\section{DISCUSSÃO}

Há controvérsias na literatura quanta à associação entre sintomas da DRGE e sexo. Segundo Oliveira et al. ${ }^{3}$ alguns estudos encontraram maior risco para homens e outros para mulheres, o que pode sugerir que o sexo não é determinante na ocorrência de sintomas da doença. Já Corsi et al. ${ }^{12}$ relatam que estudos mostram a elevada prevalência da DRGE, acometendo ambos os sexos. Em relação à presença de pirose, o presente estudo evidenciou equilíbrio entre os dois sexos nos entrevistados que referiam o sintoma. A maioria dos entrevistados estavam com IMC normal. O estudo de Corsi et al. ${ }^{12}$ demonstrou não haver aparente relação entre DRGE e IMC.

No estudo de Suzuki et al., ${ }^{14}$ realizado com universitários da Faculdade de Ciências Médicas da Santa Casa de São Paulo, detectou-se a presença de pirose e/ou regurgitação uma vez ou mais por semana em apenas 15,9\% dos entrevistados. Já nos estudantes de medicina da UFT, a frequência encontrada neste mesmo tipo de análise foi de 47,3\%. A casuística do segundo superior à do primeiro pode sugerir que os estudantes da UFT estão mais expostos a fatores agravantes quando comparados aos universitários do estudo citado.

Apesar de não confirmado o diagnóstico da doença nos estudantes, a presença de pirose e/ou regurgitação ácida são aceitas como marcadores clínicos da DRGE, quando ocorrerem duas vezes por semana no período das últimas quatro a oito semanas, segundo o Consenso Brasileiro para a DRGE. ${ }^{10}$ Sendo assim, pode-se considerar que os estudantes de medicina da UFT que afirmaram ter pirose mais de uma vez por semana tinham alta probabilidade de apresentar a DRGE, ou seja, 35 (18,8\%, IC95\% 13,5\% a 25,2\%). Essa prevalência foi inferior à encontrada no estudo de Oliveira et al. $31,3 \% .^{3}$

No estudo de Moraes-filho et al. ${ }^{8}$ foi constatada uma relação positiva entre sintomas da DRGE e ingestão de alimentos gordurosos. No presente estudo, a maioria $(63,8 \%)$ relatou aumentar a queimação com a ingestão desse tipo de alimentos. Isso é coerente com a afirmação de que a gordura retarda o esvaziamento gástrico e se associa ao refluxo gastroesofágico, do estudo de McCallum et al. ${ }^{13}$

Os achados relacionados a aspectos psicológicos confirmam a grande importância desses fatores na determinação da DRGE, segundo estudo realizado por Oliveira et al. ${ }^{3}$ No presente estudo constatou-se um agravo do sintoma pirose na presença de fatores emocionais em $67,5 \%$ dos estudantes. 
Grande parte (97,8\%) dos estudantes não é tabagista, por isso não foi possível verificar a presença de associação entre tabagismo e o agravamento do sintoma.

O tratamento clínico visa aliviar os sintomas, cicatrizar as lesões e prevenir recidivas e complicações, porém o grande problema deste tipo de tratamento não é controlar os sintomas em curto prazo, mas sim manter os pacientes assintomáticos a longo prazo. ${ }^{1} \mathrm{~A}$ minoria dos entrevistados utilizava medicações para alívio rápido após o surgimento dos sintomas, mostrando a preferência por métodos não medicamentosos ou até mesmo pela resolução espontânea, sugerindo que os sintomas fossem leves e passageiros. Entretanto, uma vez que esses fatores agravam a ocorrência do sintoma esofágico, pode-se inferir que nos indivíduos com DRGE eles aumentem o risco de esofagite, devendo por isso ser evitados.

Conclui-se que os estudantes de medicina em estudo apresentaram uma alta prevalência do sintoma pirose. A ocorrência da pirose pelo menos uma vez por semana foi relatada pela maioria dos entrevistados, tendo uma frequência maior quando comparada a de outros estudos semelhantes. O sintoma era agravado por ingestão de alimentos gordurosos ou condimentados, alterações emocionais e bebidas alcoólicas.

\section{REFERÊNCIAS}

1. Nasi A, Moraes-Filho JPP, Cecconello I. Gastroesophageal reflux disease: an overview. Arq Gastroenterol. 2006;43(4):334-41

2. Guerra MTPM, Abreu BAL. Doença do refluxo gastroesofágico: classificação cintilográfica. Rev Bras Clin Med. 2010;8:10-4.

3. Oliveira SS, Santos IS, Silva JFP, Machado EC. Prevalência e fatores associados à doença do refluxo gastroesofágico. Rev Arq Gastroenterol. 2005;42(2):116-21.

4. Federação Brasileira de Gastroenterologia. Projeto Diretrizes. Associação Médica Brasileira e Conselho Federal de Medicina. Refluxo Gastroesofágico: Diagnóstico e Tratamento. p. 1-18.[Internet].São Paulo; 2003 [updated 2003 Oct; cited 2014 Jun]. Available from: http:// www.projetodiretrizes.org.br/projeto_diretrizes.php

5. Moraes-Filho JPP, Domingues G. Gastroesophageal reflux disease. Rev Bras Med. 2009;66(9):303-10.

6. Wahlqvist P.Symptoms of gastroesophageal reflux disease, perceived productivity, and health-related quality of life. Am J Gastroenterol. 2001;96(8 Suppl):S57-61.

7. Barbuti RC, Moraes-Filho JPP. Gastroesophageal disease. Rev Bras Med. 2010;67(12):67-75.

8. Moraes-Filho JPP, Chinzon D, Eisig JN, Hashimoto CL, Zaterka S. Prevalence of heartburn and gastroesophageal reflux disease in the urban Brazilian population. Arq Gastroenterol. 2005;42(2):122-7.

9. Andreollo NA, Lopes LR, Coelho-Neto JS. Gastroesophageal reflux disease: what is the effectiveness of diagnostic tests? Arq Bras Cir Dig. 2010;23(1):6-10.

10. Barros SGS. Doença do refluxo gastroesofágico: prevalência, fatores de risco e desafios. Arq Gastroenterol. 2005;42(2):71.

11. Carine P, Nuñez MRZ, Nicoleit AR, Rodrigues DFP, Pereira SW, Galvani GC. Prevalência de sintomas de doença do refluxo gastroesofágico em pacientes com indicação de endoscopia digestiva alta. Arq Catarin Med. 2010;39(1):62-9.

12. Corsi Paulo Roberto, Gagliardi Danilo, Horn Mozar, Pochini Celso de Castro, Oliveira Neto Reinaldo Martins de. Presença de refluxo em pacientes com sintomas típicos de doença do refluxo gastroesofágico. Rev Assoc Med Bras. 2007;53(2):152-7.

13. McCallum RW, Berkowitz DM, Kerner E. O esvaziamento gástrico em pacientes com refluxo gastroesofágico. Gastroenterology 1981;80:285-91.

14. Suzuki NM, Nakae TK, Castro PC, Bonadia JCA. Doença do Refluxo Gastroesofágico (DRGE): Epidemiologia e qualidade de vida em estudantes universitários. Arq Med Hosp Fac Cienc Med Santa Casa São Paulo. 2011;56(2):65-7. 